\title{
Transnacionalidad sindical. \\ Herramienta de integración, cambio y equidad global.
}

\author{
Union transnationality. \\ Tool of integration, change and global equity.
}

\author{
José Gregorio Noroño Sánchez
}

VENEZUELA

\begin{abstract}
Resumen: El propósito de este artículo es analizar la transnacionalidad sindical como herramienta de cambio y equidad global, tomando como referencia el desarrollo en las organizaciones sindicales. La investigación es de tipo cualitativa y se asume desde un paradigma postpositivista con fundamento ontológico. Para la interpretación de los datos se utilizó el análisis hermenéutico y la heurística. Los resultados demostraron que para que exista la transnacionalidad sindical las organizaciones deben atravesar un proceso estructurado de satisfacción de condiciones.
\end{abstract}

Palabras clave: transnacionalidad sindical; mundialización; equidad global.

Abstract: The aim of the paper is to analyze trade union transnationality as a tool for global change and equity, taking as reference the development in trade union organizations. The research is qualitative and is assumed from a postpositivist paradigm with ontological foundation. For the interpretation of the data the hermeneutic analysis and the heuristic were used. The results showed that for trade union transnationality to exist, organizations must go through a structured process of satisfying conditions.

Keywords: union transnationality; globalization; global equity.

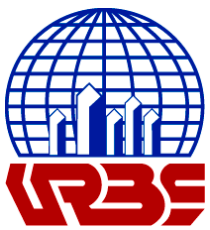

José Gregorio Noroño es Abogado, Magíster en Derecho del Trabajo, Doctor en Ciencias Gerenciales de la Universidad Rafael Belloso Chacín y participante del Postdoctorado en Integración y Desarrollo de América Latina. Es docente universitario y autor del libro "Ética Sindical y relaciones laborales: Un valor impulsor en empresas privadas", así como de diversos artículos relacionados con organizaciones sindicales, relaciones laborales y ética.

Contacto: jnorono@urbe.edu.ve - ORCID: https://orcid.org/0000-0001-97772733 
Transnacionalidade da união.

Ferramenta da integração, mudança e equidade global

Resumo: O objetivo do artigo é analisar a transnacionalidade sindical como instrumento de mudança e equidade global, tomando como referência o desenvolvimento das organizações sindicais. A pesquisa é qualitativa e é assumida a partir de um paradigma póspositivista de fundamentação ontológica. Para a interpretação dos dados, utilizou-se a análise hermenêutica e a heurística. Os resultados mostraram que para que a transnacionalidade sindical exista, as organizações devem passar por um processo estruturado de satisfação de condições.

Palavras-chave: Transnacionalidade da união; globalização; equidade global.

\section{Introducción}

a realidad global ha sobrepasado todos los niveles previstos, se presentan un sinfín de eventos propiciados por la mundialización que antes era de imposible concepción. Transmisiones en tiempo real, intercambio comercial intercontinental virtual, adopción de términos lingüísticos internacionales y políticas sociales de similares aplicabilidades. Estamos en presencia de un fenómeno globalizador sin precedentes, debido al alcance de las redes sociales y las nuevas tecnologías de la información y la comunicación (TIC). Sin embargo, esa realidad aún no ha trastocado el bienestar laboral mundial, y ese debe ser el próximo paso: convertir los beneficios laborales en condiciones inclusivas y de escala mundial.

De acuerdo con Añez y Bonomie (2007), el efecto de la globalización ha generado consecuencias negativas en las condiciones laborales y significado la entrada de políticas de flexibilización que se han traducido en la pérdida de beneficios laborales, precariedad y disminución de ingresos para los trabajadores. Sin embargo, las organizaciones sindicales, aun con los dilemas éticos que persisten en su organización, han hecho frente a estas políticas, en algunos casos de forma exitosa y en otros no tanto. Es por esto que cobra relevancia el siguiente interrogante: ¿Se necesitan organizaciones sindicales transnacionales para equipararse con la realidad global? Y de resultar cierta, ¿Cuáles son los retos de los sindicatos para responder a las consecuencias derivadas del fenómeno de la mundialización?

\section{La realidad empresarial}

Las empresas en la actualidad se caracterizan por la expansión de los procesos de externalización y por la mutación a formas no convencionales de generar rentabilidad, valor y ser sustentables, abriéndose a nuevos 
mercados gracias a la globalización y la liberación de las fronteras entre países.

La organización del trabajo es diferente en el mundo, por eso, cada país tiene una legislación laboral de acuerdo con su realidad. Unificar las legislaciones laborales implicaría una serie de acuerdos en temas de integración regional e internacional, los cuales, en el caso de América Latina, aún no se han definido del todo. Existen mecanismos multilaterales de integración, sin embargo, no se han establecido las normas mínimas de movilidad migratoria, que, a la vez, permitan la autonomía y soberanía de cada país.

Las organizaciones multilaterales como la Comunidad de Estados Latinoamericanos y Caribeños (CELAC), la Alianza Bolivariana para los Pueblos de Nuestra América (ALBA) y el Mercado Común del Sur (MERCOSUR), destinadas a la cooperación e integración de la región, no han desarrollado una legislación laboral común, ni la protección de los trabajadores migrantes en ninguno de los países miembro.

Por su parte, Miravert (2009) establece que en Europa, específicamente en España, los sindicatos no solo han actuado como organizaciones de defensa y promoción de los intereses y derechos de los trabajadores migrantes, sino también como instancias de intervención diversificada en el proceso de integración, e incluso como primer espacio de inserción en el país de acogida. Las organizaciones sindicales latinoamericanas deben estar formadas y preparadas para dar este salto en su visión proteccionista. Se debe entender que en muchos lugares del mundo la palabra migrante significa mano de obra barata, sin protecciones legales y beneficios de ninguna especie.

Es importante reconocer el papel protagónico que desempeñan las organizaciones sindicales en el futuro escenario latinoamericano, debido al espectro de las alianzas integracionistas que se instalan como alternativas para diversificar las economías y la participación cada vez más significativa de la región en la aldea global. Las organizaciones sindicales de Latinoamérica deben modernizarse, fusionarse y adaptarse a los nuevos contextos, buscando convertirse en una fuerza laboral transnacional, que se equipare al reto globalizador. Para este propósito podría seguirse las actividades sindicales de Europa como referente para Latinoamérica (Miravert, 2009).

\section{El reto sindical transnacional}

Un reto del sindicato transnacional es la ruptura de las barreras lingüísticas. En Latinoamérica y el Caribe convergen al menos cinco (5) idiomas, entre 
ellos, el español, portugués brasileño, inglés de Guyana, francés de algunas islas del Caribe y el papiamento. Aunque ya hay herramientas de traducción en vivo, como las ofrecidas gratuitamente en la web, existen diferentes de aplicaciones que pueden ser importantes para expandir las fronteras de las organizaciones sindicales. Sin embargo, estas herramientas jamás sustituirán la facilidad de comunicarse sin intermediarios, ya sean humanos o tecnológicos. Las organizaciones sindicales que intenten hacer el salto a la transnacionalidad deben sopesar y hacer frente a las barreras lingüísticas.

En el siguiente mapa se presentan las lenguas que existen en América, para ilustrar el reto que tienen las organizaciones sindicales ante el tránsito a la transnacionalidad.

Mapa 1. Idiomas en América

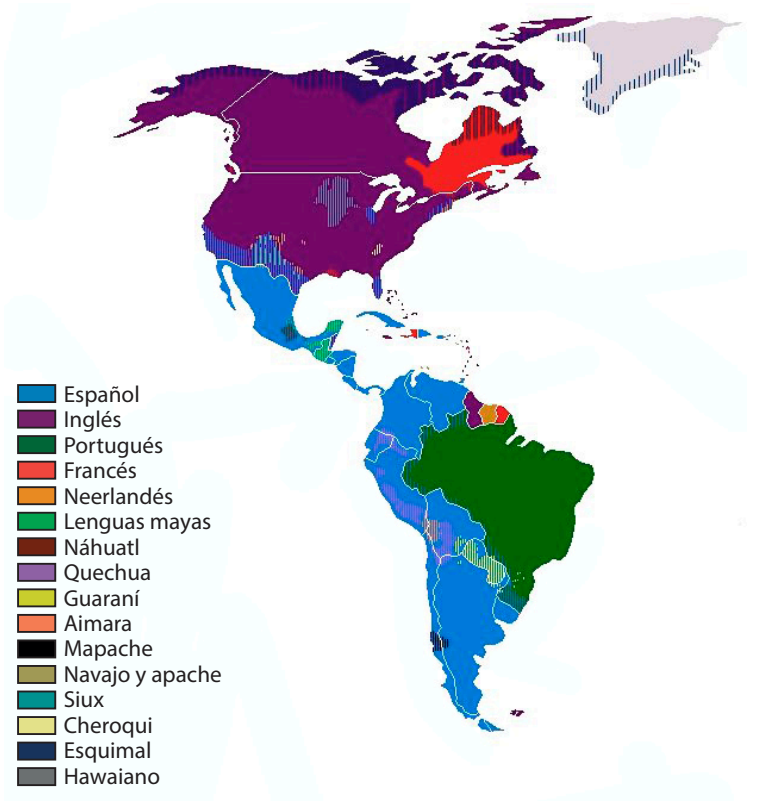

Fuente: Saber es práctico.

Tales consideraciones responden al orden descriptivo y disruptivo que debe imperar en las organizaciones sindicales modernas, actualizadas y transnacionales que deben aparecer en los próximos años en Latinoamérica para el beneficio de los trabajadores. Se han empezado a consolidar alternativas claras de fusión sindical, que buscan la consolidación nacional e internacional de las fuerzas laborales para lograr la equidad global. 
Una vez se supere el tema de legislación regional y las barreras lingüísticas, se debe establecer un plan de mundialización de beneficios derivados de las convenciones colectivas, ya sean a nivel latinoamericano, continental o mundial, con el propósito de que las actividades laborales realizadas por cada empresa transnacional tengan las mismas condiciones en todo el continente, es decir, que el trabajo realizado por un montacarguista de una planta embotelladora de Méjico, sea el mismo al de un montacarguista de la misma empresa en Barranquilla, Colombia.

Estos retos serán una utopía si no se consolidan los pasos previos, pues la construcción de un sindicato global transnacional es solo posible invirtiendo en su formación, reestructuración, y adaptándolo a las nuevas tendencias. Es importante, además, estudiar los modelos sindicales europeos, de ahí que una opción es hacer benchmarking de estos modelos como referentes para consolidar las organizaciones sindicales en el contexto latinoamericano.

Cumplir con el "Reto Americano" solo es posible a través de organizaciones sindicales de cuarto o quinto grado de nivel de desarrollo, fusión y progreso. Se entiende por organismo sindical de cuarto grado aquella que posee afiliados solo en su continente, o las llamadas organizaciones sindicales continentales; por otra parte, las organizaciones sindicales de quinto grado son las organizaciones sindicales mundiales, ya que poseen afiliados dentro y fuera del continente.

Siendo estas organizaciones aglutinadoras de sindicatos de tercer grado, las cuales son denominadas confederaciones, al fusionar tres federaciones dentro de una nación, se destaca la ardua labor que tienen por delante los organismos de representación colectiva. La actividad sindical en América Latina es relativamente reciente, su desarrollo cada vez debe ser más acelerado si pretende estar a la par con el resto del mundo y en consonancia con el fenómeno de la globalización.

Son escasos los esfuerzos conocidos en la región para generar alianzas vitales para los intereses de los trabajadores del continente o en su defecto para el trabajador latinoamericano. Se registran pocos intentos de fusión o creación de sindicatos transnacionales. En países como Colombia, algunas federaciones se están fusionando para formalizar organizaciones nacionales, con miras al fortalecimiento del trabajador en su territorio, pero hace falta dar el paso internacional, y para ello se necesitan acercamientos para incluir las dimensiones humana, tecnológica, material y financiera.

La Organización Internacional del Trabajo (OIT) (2018: 316) se define como la organización responsable de contribuir a la aplicación efectiva de los principios generales de la libertad sindical, que constituye una de las 
garantías primordiales para la paz y la justicia social. Su función consiste en garantizar y promover el derecho de organización de los trabajadores y de los empleadores, lo cual también incluye a las de orden internacional.

La OIT ha recibido, solo en el año 2017, tres mil trescientas diez (3310) demandas de prácticas antisindicales de organizaciones sindicales, por parte de los diferentes gobiernos del mundo, con lo cual se observa que existen alternativas tangibles para el buen funcionamiento de los sindicatos transnacionales, que se avizoran para dar respuesta similar al fenómeno de la globalización y que, al mismo tiempo, un organismo de representación colectiva puede ser más efectivo mientras más sólido sea.

La solidaridad sindical internacional constituye uno de los objetivos básicos de todo movimiento sindical y ha inspirado la norma contenida en el artículo 5 del Convenio número 87, según la cual toda organización, federación o confederación tiene el derecho de afiliarse a organizaciones internacionales de trabajadores. Los sindicatos y federaciones deben ser libres para afiliarse a aquellas federaciones o confederaciones internacionales que estimen convenientes sin de sus respectivos gobiernos (OIT, 2018: 198).

La OIT (2018: 199) señala varias consideraciones relativas al artículo 5 del Convenio número 87. Como lo indican los trabajos preparatorios del instrumento, de la solidaridad de intereses que une a los trabajadores, por una parte, y a los empleadores, por otra; esta solidaridad no se limita ni a una empresa ni a un ramo de actividad determinados, ni siquiera a la economía nacional, sino que se extiende a la economía internacional entera. Este derecho corresponde a la práctica seguida por la Organización de las Naciones Unidas y la OIT, al haber reconocido ambas formalmente, las organizaciones internacionales de trabajadores y empleadores asociándolas directamente a sus trabajos.

Un sindicato transnacional está cobijado por la legislación internacional que avala su fundación, acción, legitimación y protección por parte de organismos multilaterales que coadyuvan a la concreción de sus principios rectores, como la protección al trabajo como hecho social internacional y su función de garante del cumplimiento de beneficios logrados a través de la celebración de convenciones colectivas de índole internacional. Con lo cual se establece el respaldo y la confianza de mejores resultados a la hora de intentar modificar la calidad de vida de sus miembros en las organizaciones sindicales transnacionales.

Los organismos continentales y mundiales solo pueden afiliar o fusionarse con organizaciones de segundo o tercer grado, es decir, pueden afiliar a federaciones o confederaciones de trabajadores de cada país que así lo 
solicite, pero no pueden afiliar a personas o individuos, así como tampoco a sindicatos de primer grado. Cabe destacar que el otro reto evidente es la celebración de convenciones colectivas a nivel continental y mundial, con lo cual se estaría en presencia de un organismo que puede hacer frente a la tendencia global de las empresas multinacionales y nivelar los beneficios a escala internacional.

Otro factor decisivo depende, en gran medida, del desarrollo sindical y la madurez de los líderes de los proyectos sindicales transnacionales, en el sentido de que el propósito es brindar una respuesta acorde con el fenómeno de la globalización. Para Noroño y Seijo (2016), la organización sindical es el único instrumento jurídico avalado internacionalmente para representar a los trabajadores y celebrar acuerdos que permitan optimizar su calidad de vida. Es una ardua tarea, un gran reto para las organizaciones sindicales modernas y un gran desafío para afrontar con éxito la nueva modalidad del trabajo en el siglo XXI, caracterizado por el avance tecnológico y de las telecomunicaciones.

\section{Necesidades globales}

De acuerdo con João Felício (2006), es necesario establecer una respuesta integracionista dadas las intenciones de enarbolar alianzas semejantes al Área de Libre Comercio de las Américas (ALCA) propuesto por Estados Unidos, con el respaldo de sus aliados regionales, lo cual, en su criterio, pone en evidencia las necesidades de integración regional sindical. Hace falta agilizar lo que él denomina "la agenda del sur", que no es más que la acción combinada del factor social latinoamericano y las organizaciones sindicales. Sin embargo, acota Felício (2006), la idea de integración regional comercial no responde a una mirada interna hacia el continente sureño, sino al intento neoliberal de transformación de los países latinoamericanos en un apéndice de la economía norteamericana. No se parte de las necesidades de los pueblos, sino del programa neoliberal y los intereses del capital.

Las respuestas integracionistas actuales entre ellas la Comunidad Andina de Naciones (CAN) y más recientemente el Mercado Común del Sur (Mercosur) no responden a los pueblos involucrados, sino a la orientación estratégica de una nueva forma neoliberal; no obstante, son una oportunidad para construir otras formas de integración regional (Felício, 2006). En este sentido, hay que pasar de las propuestas a la acción, materializar las alianzas sindicalistas, de ahí que:

(...) reciente fundación de la nueva Confederación Sindical Internacional (CSI), fruto de la fusión de dos centrales mundiales, la fusión de la ORIT (Organización Regional Interamericana de 
Trabajadores) y de la CLAT (Confederación Latinoamericana de Trabajadores) de la cual deberá surgir una nueva central continental. (...) como parte de esta misma estrategia, se viene impulsando la realización de foros sindicales de las Américas (el primero, durante, la Cumbreen Mardel Plata en noviembre de 2005, el segundo, durante el Foro Social Mundial en Caracas, en enero de 2006, y el tercero se llevó a cabo en Cochabamba en diciembre de 2006) que convocados por la ORIT cuentan con la participación activa de centrales sindicales de todas las orientaciones políticoideológicas de nuestro continente, sin exclusiones: un caso singular de experiencia unitaria en el sindicalismo mundial, que no tiene paralelo en otro continente (Felício, 2006).

Se puede cambiar la escena mundial laboral a través de esta clase de organizaciones que buscan defender los derechos de los trabajadores, incrementar los beneficios y mejorar las condiciones laborales y calidad de vida de los trabajadores. En otras palabras, es establecer el trabajo decente como norte, de la mano de la OIT.

En noviembre de 2006, se concretó la disolución de la Confederación internacional de Organizaciones Sindicales Libres (CIOSL) y de la Confederación Mundial del Trabajo (CMT) para fundar y fusionarse en la Confederación Sindical Internacional (CSI), la central sindical más grande y representativa del mundo con cerca de 166 millones de miembros afiliados a 309 organizaciones sindicales en más de 156 países. Su sede es en Bruselas, Bélgica, y ostenta la Secretaria General Sharon Burrow, de procedencia australiana, y como presidente Joao Antonio Felício de Brasil.

La CSI ha definido sus prioridades y metas estableciendo la coherencia global, la gobernanza mundial de migración, la organización, la identificación de países en riesgo laboral y el respeto y respaldo a los trabajadores del hogar, como sus principales ejes temáticos y bastiones de enfoque mundial. Lo cual es un avance importante, pero no representan un cambio e impacto internacional.

Una única central mundial no basta para hacer frente al proceso globalizador, hace falta mayor empeño y alianzas para generar los aportes sindicales que se necesitan. Se debe seguir apostando a tales organismos de representación colectiva para aspirar a una escena laboral internacional diferente. El movimiento sindical transnacional no debe ser conformista, al contrario, debe seguir su ruta hacia la diversidad y la conformación de otras centrales internacionales que sigan defendiendo los derechos de los trabajadores. 


\section{Taxonomía sindical}

Cuando se piensa en las exigencias humanas, es imposible pasar desapercibida la tesis de Maslow (2005) sobre la jerarquía de las necesidades. Su teoría piramidal ejemplificadora resulta importante para este estudio, pues hacer un acercamiento a esas necesidades permite contrastar los objetivos sindicales transnacionales. Maslow (2005) desarrolló una jerarquía piramidal que describe los escalones que posee una persona y que se pueden adaptar al caso sindical. Señala que, una vez los individuos tienen satisfechas ciertas condiciones como nutrición, cobijo y seguridad vital, tratan de satisfacer otras necesidades y así se escala entre cada una de ellas, las cuales se ejemplifican en la siguiente figura.

Figura 1. Teoría de las Necesidades de Maslow

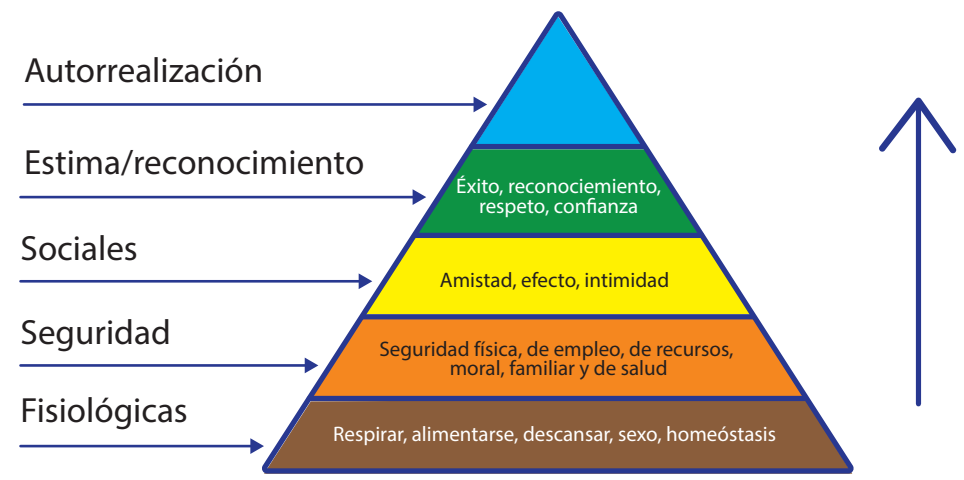

Fuente: J. Finkelstein

A nivel sindical se pueden establecer relaciones con la teoría de las necesidades de Maslow (2005). En primer lugar, las organizaciones sindicales nacen en un contexto capitalista y, por norma general, dan los primeros pasos en una empresa donde se presta servicio, siendo su fisiología creativa la base de tal pirámide y en la que los líderes sindicales empiezan a adquirir las cualidades de liderazgo necesarias para afrontar las responsabilidades de defensa de los derechos laborales de sus afiliados.

En segundo lugar, las organizaciones deben exigir reformas laborales a través de la presentación de pliegos de peticiones y convenciones colectivas, que, al ser efectivas, generan la satisfacción de sus asociados y elevación de su confianza hacia el grupo de representantes sindicales. Tal actividad es vital si se desea seguir escalando en reputación, liderazgo y eficacia sindical. 
En tercer lugar, una vez que el organismo de representación ha demostrado las habilidades y cualidades de liderazgo y ha contribuido a mejorar la calidad de vida de sus agremiados, aparece la necesidad sindical de expansión, ya sea para sindicalizar a los demás trabajadores de la empresa, del estado, entidad federal, departamento o los del mismo arte, oficio o profesión de su país.

Cumplidas estas primera tres etapas, tanto las organizaciones sindicales como sus principales líderes suelen recibir un reconocimiento por su labor, que, a diferencia de la teoría de Maslow, es un reconocimiento por la reputación y conducta ética. En tal sentido, estos ingredientes se conjugan para impulsar las organizaciones de cuarto grado o también denominados sindicatos continentales. Con base en el arduo trabajo, la consecución de objetivos laborales y una entrega acorde a los beneficios de sus agremiados, una organización sindical llega a la cúspide de la pirámide.

Una organización sindical de cuarto grado alcanza la fase más alta de satisfacción de las necesidades sindicales, cuando, por medio de la fusión o fundación de una nueva organización sindical, da el paso hacia la representación mundial de la clase trabajadora, convirtiéndose en una organización sindical de quinto grado, último grado existente y reconocido para una organización de representación colectiva. Es en este escenario, donde las organizaciones de trabajadores logran el más alto grado de integración, fusión, cohesión y compromiso, que se necesitan muchas más organizaciones sindicales transnacionales, con las cuales se busque integrar los salarios a nivel internacional y se persiga la equidad en un mundo lleno de desigualdades globales.

\section{Resultados}

La técnica ontológica exige la conceptualización sistematizada de los aspectos relacionados con los estudios. En este sentido, Velásquez (2010) señala que tal descripción demanda aspectos metodológicos para su desarrollo, explorando diversas herramientas para acceder al conocimiento acumulado y empírico. Para los fines de esta investigación, las precisiones conceptuales se presentan a continuación.

Se parte de la figura del sindicato de primer grado, siendo este la mínima expresión de una organización de representación colectiva. Por lo general, está compuesto por 20 o 25 trabajadores, y se denomina sindicato de empresa o entidad de trabajo. Puede tener ámbitos locales y en empresas con más de una sucursal o dependencia, y aplicar a todo un departamento o estado; en algunos casos, el ámbito puede modificarse y convertirse en sindicato de empresa con proyección regional o nacional en las legislaciones que así lo permiten. 
El sindicato es la agrupación de trabajadores bajo dependencia de un patrono, empleador o jefe, en una empresa, explotación o faena, que articula la conformación de un instrumento constitutivo que brinda la posibilidad de realizar actos jurídicos en representación de sus integrantes, con el propósito de buscar, de manera progresiva, beneficios derivados de la relación de trabajo. Tal agrupación de intereses y finalidades derivan en la constitución de un organismo sindical que persigue la protección, desarrollo y vigilancia de los derechos y garantías laborales.

Existen en todos los ordenamientos jurídicos latinoamericanos las agrupaciones sindicales de segundo grado, compuestas por la fusión de organismos sindicales de primer grado, es decir, cinco o más sindicatos de primer grado pueden acordar la cohesión de fuerzas y principios de sus agremiados para fundar federaciones de trabajadores, las cuales tienen impedido afiliar a trabajadores, y solo pueden afiliar otros organismos sindicales de su misma rama, especialidad, profesión o especie.

Las federaciones pueden ser de ámbito regional o nacional, dependiendo del propósito y la distribución geográfica de los sindicatos participantes. Estas formas de organización representan una conciencia de superación de necesidades y requieren de una visión amplia que represente los intereses colectivos. Por norma general, representan y agrupan a la mayoría de los trabajadores de un sector específico de trabajadores.

Las organizaciones sindicales de tercer grado son las confederaciones, las cuales afilian, fusionan o aglutinan otras federaciones de diferentes ramas, industrias, actividades y ámbitos. Responden a sus afiliados por los asuntos nacionales y se estructuran de forma autónoma en la taxonomía interna, de propósitos o de visión prospectiva. Las confederaciones son las máximas organizaciones sindicales de un país, las cuales cuentan con un proceso de legalidad administrativa que le permite a sus afiliados celebrar actos de representación. Si bien persiguen intereses comunes, en cada ordenamiento jurídico se estipulan obligaciones y deberes para cada sindicato sin menoscabo de su grado.

En orden de estructura y cumplimiento con la teoría de las necesidades de Maslow, un órgano sindical de tercer grado puede afiliarse a una central de cuarto grado o centrales continentales. Estas figuras internacionales representan a las confederaciones en cada continente, y su objetivo es proteger las relaciones laborales de sus agremiados dentro de su respectivo ámbito espacial. Es decir, se evidencia un derecho internacional para estas organizaciones con el fin de darle continuidad al desarrollo sindical del continente. 
Las organizaciones sindicales de quinto grado están compuestas por dos o más organizaciones continentales, en las cuales configuran los sindicatos mundiales. Estos organismos representan la cúspide sindical mundial y es una forma de hacerle frente al fenómeno de la globalización, en virtud de la extensión del amparo a los trabajadores. Llama la atención que solo exista una organización sindical de ámbito mundial, de ahí que este texto prioriza la formulación de una respuesta acorde con los aspectos transnacionales propios de los organismos de representación colectiva de ámbitos mundiales.

\section{La justicia y equidad global}

La equidad, la justicia social y la progresividad de los beneficios laborales se enriquece y fortalece cuando las organizaciones sindicales están enfocadas en lograr las reivindicaciones para sus agremiados. De acuerdo con Noroño y Seijo (2016) son la fuente de consecución del principio de progresividad $y$, en tal sentido, la OIT respalda las acciones que persigan los logros de la clase trabajadora mundial.

Las organizaciones mundiales de representación colectiva laboral dan origen a procesos de integración local, regional e internacional, es por eso que existe la posibilidad de articular mecanismos que propendan por una justicia para los trabajadores.

Las organizaciones sindicales asumen roles protagónicos en sus comunidades, en sus países y en la escena mundial, pero para que exista equidad global deben existir representantes de los trabajadores en todo ámbito. Se debe equiparar el sindicalismo mundial al tamaño del capital, al menos en su estructura organizativa, y a la visión global que hoy es de fácil acceso gracias a las TIC. Sin embargo, existen retos y caminos por recorrer en el devenir sindical, que encauzarán mejores vientos para los trabajadores a nivel mundial.

\section{Conclusiones}

La internacionalización económica derivada de la globalización y la expansión y desarrollo tecnológico y comunicacional ha conllevado a la respuesta de las organizaciones sindicales. Autores como Etchemendy y Berins (2008) son optimistas y señalan que la activación sindical responde a la condición del mercado de trabajo y a la estrategia política. Mientras mejores sean las ofertas de trabajo y generación de ingresos empresariales, mejores oportunidades para los organismos sindicales. En este sentido, las organizaciones sindicales deben perseverar y organizarse de forma continental y mundial. 
De esta manera, también se puede dar una respuesta a otro fenómeno social de escala mundial, como la migración. Las organizaciones sindicales de quinto grado pueden defender los derechos de forma integral del trabajador, haciendo frente a los mecanismos de flexibilización o diminución de los beneficios como fundamento en la procedencia del individuo, o condición legal en el país de acogida.

Un fundamento para redoblar los esfuerzos para la creación de organismos sindicales transnacionales es la búsqueda de la equidad e igualdad laboral en un mundo que, según Viteri (2016), presenta la mayor cantidad de condiciones desfavorables referidas a la igualdad laboral sin discriminación. Un organismo sindical transnacional es una respuesta simétrica a las asimetrías y distorsiones que causa el capital bajo la racionalidad de la rentabilidad y sustentabilidad del negocio en detrimento del bienestar general y de los derechos laborales. Un sindicato transnacional es una fuente de equidad global y una herramienta poderosa para el desarrollo mundial.

\section{Referencias bibliográficas}

1. AÑEZ, Carmen y BONOMIE, María. (2007). Relaciones laborales y sindicatos en el contexto adverso de la globalización. Revista Venezolana de Gerencia, Vol. 12, N ${ }^{\circ}$ 37. Pp. 50-65.

2. ETCHEMENDY, Sebastián y BERINS, Ruth. (2008). Golpeados pero de pie. Resurgimiento sindical y neocorporativismo segmentado en Argentina (20032007). Revista POSTData: Revista de Reflexión y Análisis Político, № 13. Pp. 142-192.

3. FELÍCIO, Joao Antonio. (2006). El movimiento sindical a la hora de la integración regional. Disponible en Internet: https://bit.ly/2NGnyfF Consultado: 5.04.2018

4. MASLOW, Abraham. (2005). El management según Maslow: una visión humanista para la empresa de hoy. Barcelona: Editorial Paidós Ibérica. 347 pp.

5. MIRAVERT, Pablo. (2009). Trabajadores inmigrantes, sindicatos y participación. Valencia: Universidad de Valencia.

6. NOROÑO, José Gregorio. (2014). Retos y perspectivas de las organizaciones sindicales en el contexto de la globalización de las relaciones laborales en Venezuela. Revista Lex Laboro, Vol. VI. Pp 33-53.

7. NOROÑO, José Gregorio y SEIJO, Cristina. (2016). Ética Sindical y Relaciones Laborales: Un valor impulsor en empresas privadas. Madrid: Publicia. 388 pp.

8. OIT Oficina Internacional del Trabajo (2018). Libertad Sindical. Recopilación de decisiones del Comité de Libertad Sindical. Ginebra: OIT. 


\section{8}

9. VELÁSQUEZ, Torcoroma, PUENTES, Andrés Mauricio y GUZMÁN, Jaime Alberto. (2010). Ontologías: una técnica de representación de conocimiento. Revista Avances en Sistemas e Informática, Vol. 8, № 2. Pp. 211-216.

10. VITERI, Digna Marcela, PEÑAHERRERA, Pablo Guillermo y VINUEZA, Jenny Lolita. (2016). La equidad de género como parte de la responsabilidad social en el mercado laboral de la industria turística del Ecuador. Revista Ibero-Americana de Estratégia, Vol. 16, № 4. Pp. 23-36.

Para citar este artículo:

NOROÑO, José Gregorio. (2019). Transnacionalidad sindical. Herramienta de integración, cambio y equidad global. Teuken Bidikay, Vol. 10, № 15. Pp. 225238. Medellín: Politécnico Colombiano. doi:10.33571/teuken.v10n15a10

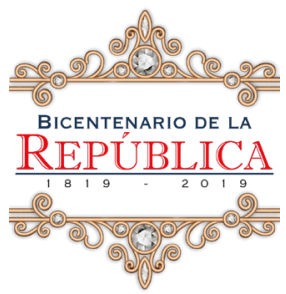

Villa de Leyva

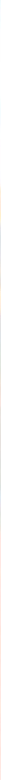

